\title{
Híbridos de Cebolla Roja (Allium cepa L.) Propagados por Bulbillos con Diferentes Diámetros Ecuatoriales, en Zona Árida
}

\author{
Onion Red Hybrids (Allium cepa L.) Propagated by Bulbillos \\ With Different Equatorial Diameters, in Arid Zone
}

Ronald Vasquez ${ }^{1}$, Berenice Zuloaga ${ }^{2}$, Fernando Gómez ${ }^{3}$, Omar Zeballos ${ }^{4}$, Alberto Anculle-Arenas ${ }^{5}$

\section{RESUMEN}

Los objetivos del presente estudio fueron determinar el mejor híbrido (Sivan $\mathrm{F}_{1}$, Pantera Rosa $\mathrm{F}_{1} \mathrm{y}$ Sevillana $\mathrm{F}_{1}$ ); el mejor diámetro ecuatorial de bulbillo (1,5 a menos de $2,0 \mathrm{~cm} ; 2,0$ a menos de 2,5 $\mathrm{cm}$ y 2,5 a menos de 3,0 cm); y la mejor combinación, para la producción comercial de cebolla, en la Irrigación Majes, Arequipa. Se empleó el diseño Bloques Completos al Azar con Arreglo Factorial 3x3. Los híbridos Sivan $\mathrm{F}_{1}$ y Pantera Rosa $\mathrm{F}_{1}$ tuvieron los rendimientos comerciales más altos $(100,4$ y $90,5 \mathrm{t}$ $\mathrm{ha}^{-1}$, respectivamente) con diferencias estadísticas significativas con respecto a Sevillana $\left(64,8 \mathrm{t} \mathrm{ha}^{-1}\right)$. Para diámetro ecuatorial de bulbillo los rendimientos fueron de 83,7; 76,3 y 63,1 $\mathrm{t} \mathrm{ha}^{-1}$, para los diámetros de 1,5 a 2,0 cm; 2,0 a 2,5 cm y 2,5 a 3,0 cm, respectivamente, sin diferencias estadísticas. Los bulbillos con diámetro de 1,5 a menos de $2.0 \mathrm{~cm}$ dieron lugar a bulbos más grandes, menor porcentaje de bulbos múltiples, mayor rendimiento de calidad "primera" y mayor cantidad de bulbos enteros comerciales. La mejor combinación resultó ser Sivan $\mathrm{F}_{1}$ con bulbillos de 1,5 a menos de 2,0 cm de diámetro ecuatorial con un rendimiento comercial de 98,4 t ha $^{-1}$

Palabras claves: Cebolla roja, bulbillos, zona árida.

\section{ABSTRACT}

The objectives of the present study were to determine the best hybrid (Sivan $F_{1}$, Pantera Rosa $F_{1}$ and Sevillana $\mathrm{F}_{1}$ ); the best onion sets equatorial diameter ( 1,5 to less than $2,0 \mathrm{~cm} ; 2,0$ to less than $2,5 \mathrm{~cm}$ and 2,5 to less than 3,0 cm); and the best combination, for the commercial production of onions, in the Majes Irrigation, Arequipa. The Random Complete Blocks design with 3x3 Factorial Arrangement was used. The Sivan $F_{1}$ and Pantera Rosa $F_{1}$ hybrids had the highest commercial yields $\left(100,4\right.$ and 90,5 $t$ ha $^{-1}$, respectively) with significant statistical differences with respect to Sevillana $\left(64,8 \mathrm{tha}^{-1}\right)$. For onions sets equatorial diameter the yields were 83,$7 ; 76,3$ and $63,1 \mathrm{tha}^{-1}$, for diameters of 1,5 to $2,0 \mathrm{~cm} ; 2,0$ to $2,5 \mathrm{~cm}$ and 2,5 to $3,0 \mathrm{~cm}$, respectively, without statistical differences. Onions sets with a diameter of 1,5 to less than 2,0 cm gave rise to larger bulbs, lower percentage of multiple bulbs, higher yield of "first" quality and greater quantity of commercial whole bulbs. The best combination turned out to be Sivan $F_{1}$ with bulbils from 1,5 to less than $2,0 \mathrm{~cm}$ in equatorial diameter with a yield commercial of de $98,4 \mathrm{tha}^{-1}$

Keywords: Onion red, onion sets, arid zone.

\footnotetext{
${ }^{1,5}$ Universidad Nacional de San Agustín de Arequipa (UNSA). Arequipa. Perú.

${ }^{2}$ Instituto de Educación Superior Tecnológico Publico de La Joya . Arequipa. Perú.

${ }^{3}$ Semiagro S.A.C. Arequipa. Perú.

${ }^{4}$ Universidad Nacional de Jaén (UNJ). Jaén. Perú.
} 


\section{INTRODUCCIÓN}

La producción nacional de cebollas rojas se orienta principalmente al mercado interno y muy poco para la exportación a países vecinos como Ecuador y Bolivia. En Perú, Ecuador y Bolivia la cebolla roja se utiliza como saborizante en las comidas, lo cual es conocido y señalado también por Saraswathi (2017) en el sentido que es una importante hortaliza y forma parte de importantes dietas, valorada por sus características aromáticas y curativas. En Arequipa y el Perú, la cebolla se cultiva por propaga por semilla botánica, para obtener plántulas en almacigueras y posterior trasplante; no se usa siembra directa, como ocurre en Europa o Estados Unidos en los que se utilizan almácigo, siembra directa y bulbillos u “onions sets" (Serra y Currah, 2002); aunque hace aproximadamente 10 años se está usando los llamados "cocos" o bulbillos, los que se producen utilizando entre 15 a $20 \mathrm{~g}$ de semilla botánica común por $\mathrm{m}^{2}$ y son cosechados entre octubre y diciembre, almacenados a temperatura ambiente por un periodo de 60 a 90 días, ser plantados entre enero a marzo y cosechados 90 días después. En Europa los bulbillos son bulbos pequeños 2 a $3 \mathrm{~g}$ de peso fresco que se obtienen sembrando semilla botánica en alta densidad (1000 a 2000 plantas $\mathrm{m}^{-2}$ ), su diámetro es menor a 25 milímetros y al plantarlos se obtienen plantas robustas con bulbos grandes y en un tiempo más corto (Serra y Currah, 2002 y Brewster, 2008). También se señala que los bulbillos (entre 8 y 21 $\mathrm{mm}$ ) son utilizados por productores pequeños y por productores especializados debido a que las plantas son más vigorosas y los bulbos se pueden cosechar hasta 8 semana antes que los de siembra directa (O'Connor, 2006). Hay varios estudios en Arequipa y en el mundo (Jordaan et al., 2017) que demuestran la factibilidad del uso de bulbillos de cebolla para la producción comercial de bulbos; algunos autores hallaron que la mejor densidad para la producción de bulbillos era de 6 a $8 \mathrm{~g}$ de semilla botánica por $\mathrm{m}^{2}$ (Alemzadeh,
2007); y al comparar los sistemas de siembra directa, trasplante y bulbillos, se halló que el trasplante tuvo el mayor rendimiento comercial $\left(29,3 \mathrm{tha}^{-1}\right)$, seguido por los bulbillos $\left(23,5 \mathrm{tha}^{-1}\right)$, siendo los bulbillos los que presentaron mayor cantidad de bulbos no comerciales (15,6 $\left.\mathrm{t} \mathrm{ha}^{-1}\right)$, $78 \%$ de bulbos partidos, $21 \%$ de floración prematura o "bolting" y mayor precocidad con 94 días de la plantación a la cosecha, demostrando la posibilidad del uso de bulbillos en la producción de bulbos (Ketema et al., 2013). El primer trabajo sobre el uso de bulbillos en Arequipa, lo realizó Málaga (1992) en cebolla 'Roja Americana' y halló una altura de parte aérea de 48,0 cm; un peso promedio de bulbo de $120 \mathrm{~g}$ y un rendimiento de $15,6 \mathrm{t} \mathrm{ha}^{-1}$; posteriormente Armejo (2006) trabajando con Sivan $F_{1}$ plantada en enero y febrero con bulbillos de menos de 20 , igual a 20 y más de $20 \mathrm{~mm}$ de diámetro ecuatorial, halló que para enero, el diámetro del bulbo varió de $3,7 \mathrm{~cm}$ (bulbillo de más de $20 \mathrm{~mm}$ ) a 4,0 y 4,2 cm para los bulbillos de menos de $20 \mathrm{y}$ $20 \mathrm{~mm}$, respectivamente; para febrero varió de 3,8 a 4,1 cm; el rendimiento de "primera", para enero fue de 4,6, 1,8 y 2,5 $\mathrm{tha}^{-1} \mathrm{y}$ en febrero de 9,9; 5,1 y $0,9 \mathrm{t} \mathrm{ha}^{-1}$, para los tres diámetros de bulbillos, respectivamente; a los 60 días después de la plantación, los bulbillos mayores de $20 \mathrm{~mm}$ tuvieron hasta 59,8\% de bulbos múltiples para enero y para febrero $67,7 \%$; hallándose que a menor diámetro de bulbillo menor cantidad de bulbos partidos; finalmente para la plantación de enero no se observó floración prematura, pero si para febrero, aunque en mínima proporción. Ninasivincha (2008) en cebolla 'Roja Camaneja', reporta a la cosecha, que los diámetros de bulbillos entre $0,5-1,0$ y 1,0-1,5 cm no presentaron bulbos múltiples pero si los bulbillos de $1,5 \mathrm{~cm}(6,6 \%)$ y de $2,0-3,0 \mathrm{~cm}(51,2 \%)$; el floración prematura se presentó solo para los bulbillos de 1,0-1,5, 1,5-2,0 y de 2,0-3,0 cm con 0,$2 ; 1,6$ y $12,2 \%$; respectivamente; el rendimiento de cebolla de "primera" fue de 15,2; 17,$6 ; 23,2$ y $27,6 \mathrm{tha}^{-1}$, respectivamente para los 
cuatro diámetros de bulbillos. Por otro lado, Tamo (2010) también con bulbillos de 'Roja Camaneja' concluyó que el diámetro de bulbillo mayor a $2 \mathrm{~cm}$ presentó los mayores porcentajes de doblado de planta, de bulbo múltiple, de floración prematura, de rendimiento de primera. También se halló que los mayores rendimientos, ingresos netos y costo beneficio se logró con bulbillos de $2 \mathrm{~cm}$ de diámetro plantados a distancias de 40x10 cm (Singh et al., 1996), y que en algunas épocas de plantación hay mayor presencia de floración prematura y el tamaño de bulbo es mayor, siendo el mejor tamaño de bulbillo de 1,9 a $2,5 \mathrm{~cm}$ de diámetro con un rendimiento de 45,4 $\mathrm{t} \mathrm{ha}^{-1}$ (Madisa, 1994). La floración prematura, los bulbos dobles y la precocidad están asociados al diámetro del bulbillo; así bulbillos de $29 \mathrm{~mm}$ de diámetro produjeron $31,1 \%$ de floración prematura; 69,4 $\%$ de bulbos dobles y un periodo vegetativo de 134 días; en cambio con los bulbillos de $17 \mathrm{~mm}$ de diámetro la producción de bulbos fue mayor $\left(26 \mathrm{t} \mathrm{ha}^{-1}\right)$ fue obtenido con el bulbillo de $17 \mathrm{~mm}$ de diámetro, y por otro lado los bulbillos con diámetros de 26 y $29 \mathrm{~mm}$ produjeron altas cantidades de bulbos no comerciales (13,9 y 14,9 $\mathrm{t} \mathrm{ha}^{-1}$, respectivamente) (Khokhar et al., 2002). Por otro lado la temperatura y el tiempo de almacenaje de los bulbillos influye en la producción, así se halló que bulbillos de $22 \mathrm{~mm}$ de diámetro almacenados por 90 días a $20^{\circ} \mathrm{C}$, no presentaron floración prematura, el peso promedio del bulbo y los rendimientos con buenos, a diferencia del almacenaje a $5^{\circ} \mathrm{C}$ por 90 días, en el que la floración prematura fue siete veces más (Khokhar et al., 2007) y el mismo autor (Khokhar, 2017) refiere que la alta temperatura $\left(20-25^{\circ} \mathrm{C}\right)$ de almacenaje de los bulbillos significa un incremento del rendimiento de bulbos, mientras temperaturas más altas $\left(25.5-31{ }^{\circ} \mathrm{C}\right)$ o menores a $0^{\circ} \mathrm{C}$ lo reducen. La fisiología de la cebolla (Khokhar, 2017) es afectada por el ambiente y el genotipo, como el fotoperiodo que juega un rol crítico en el desarrollo del bulbo y determina el comportamiento de un cultivar para una región dada, y el bulbeo es regulado más por la temperatura, grados día y luz rojo lejano que por el fotoperiodo; el tamaño de bulbo es menor a mayor densidad de plantación; el estrés por agua durante la formación y crecimiento del bulbo es más dañino que durante el crecimiento del follaje; el nitrógeno favorece el crecimiento del bulbo, pero también el exceso promueve le enviciamiento y alargamiento del periodo vegetativo; las giberelinas y el ethrel promueven el crecimiento y desarrollo de los bulbos; la floración y la formación del bulbo es regulado por los genes FT (locus T de floración), los genes antagonistas parecidos a los genes FT regulan la formación del bulbo (Khokhar, 2017). En la producción de cebollas por bulbillos es muy importante mantener condiciones ambientales favorables tanto en la etapa inicial como en la media del cultivo, para un buen contenido de metabolitos secundarios, y que las condiciones húmedas y frías favorecen el rendimiento, pero no los metabolitos (Ombódi et al., 2013). Los bulbillos también pueden ser utilizados para la producción de semilla botánica, por costos más bajos que el sistema tradicional, siendo los bulbillos de 26 a $31 \mathrm{~mm}$ de diámetro almacenados por 30 días a $7^{\circ} \mathrm{C}$ los de mayor rendimiento de semilla botánica $\left(595 \mathrm{~kg} \mathrm{ha}^{-1}\right)$ para el cultivar INIA Valenciana (Peluffo et al., 2016). Un riesgo del uso de bulbillos en la producción comercial de cebolla es que estos pueden traer consigo patógenos como Fusarium culmorun, F. graminearum, F. sambucinum; F.acuminatum, F.equiseti y Aspergillus niger trasmitidos desde la semilla botánica a los bulbillos de cebolla (Köycü y Özer, 1997) y en otro caso se reportan a varias especies de Fusarium y en un caso de halló hasta un $40 \%$ de incidencia por Fusarium en las plantas provenientes de bulbillos (Kivijarvil et al., 2017). La productividad de los bulbos de cebolla depende de la disponibilidad de agua en el suelo, 
fertilización adecuada y control de malezas; además que la vida útil de los bulbos es un rasgo genético que se puede mejorar mediante un cultivo eficiente, manejo de poscosecha $\mathrm{y}$ condiciones adecuadas de almacenamiento (Sekara et al., 2017).

\section{MATERIALES Y MÉTODOS}

\section{Lugar de ejecución}

El presente trabajo se llevó a cabo entre febrero y junio de 2013, en la Irrigación Majes, Arequipa, Perú; ubicada a $16^{\circ} 19^{\prime}$ Latitud Sur y $72^{\circ} 13^{\prime}$ Longitud Oeste, y una altitud de 1440 m.s.n.m. Las temperaturas medias mensuales variaron de 19,6 a 17,2 ${ }^{\circ} \mathrm{C}$; las horas de sol de 7,8 a 9,4 h; la humedad relativa de 76 a 55\%; y la precipitación acumulada fue de $8,4 \mathrm{~mm}$. El suelo fue franco arenoso, de buen drenaje y sin problemas de $\mathrm{pH}$ y conductividad eléctrica; al igual que el agua de riego proveniente de represa.

\section{Obtención de los bulbillos}

Para la obtención de bulbillos se sembró semilla botánica de Sivan F1, Pantera Rosa F1 y Sevillana F1, el 5 de septiembre de 2012 a una densidad de 12 g por $\mathrm{m} 2$, y se cosecharon el 5 de diciembre del mismo año. Los bulbillos cosechados se colocaron o almacenaron en un terreno seco y arenoso por un periodo de 60 días; tiempo después del cual se eliminó el follaje seco, se los seleccionó y clasificó de acuerdo al diámetro ecuatorial de los bulbillos (DEB).

\section{Conducción del experimento}

La plantación de los bulbillos se realizó el 07 de febrero de 2013, en surcos de 0,60 m de ancho y $0,12 \mathrm{~m}$ entre plantas, y con tres hileras por camellón del surco; y se enterraron a la mitad de su longitud. La fertilización fue de 150-130-180 $\mathrm{kg}$ ha-1 de nitrógeno, fósforo y potasio, respectivamente y aplicados vía sistema de goteo. El riego, manejo de insectos plagas, enfermedades y malezas fue igual que para los campos comerciales. La cosecha de los bulbos se realizó el 10 de mayo de 2013 y cuando el 10\% de cuellos (pseudotallos) estaban doblados y el curado duró dos semanas, luego se elimino el follaje seco para evaluar rendimiento y materia seca. En la tabla 1 se muestran los tratamientos en estudio.

\section{Tabla 1.}

Tratamientos en estudio

\begin{tabular}{|l|}
\hline Descripción \\
\hline 1. Sivan $\mathrm{F}_{1}$ con diámetro ecuatorial de bulbillo de $1.5 \mathrm{~cm}$ a menos de $2.0 \mathrm{~cm}$ \\
\hline 2. Sivan $\mathrm{F}_{1}$ con diámetro ecuatorial de bulbillo de $2.0 \mathrm{~cm}$ a menos de $2.5 \mathrm{~cm}$ \\
\hline 3. Sivan $\mathrm{F}_{1}$ con diámetro ecuatorial de bulbillo de $2.5 \mathrm{~cm}$ a menos de $3.0 \mathrm{~cm}$ \\
\hline 4. Pantera Rosa $\mathrm{F}_{1}$ con diámetro ecuatorial de bulbillo de $1.5 \mathrm{~cm}$ a menos de $2.0 \mathrm{~cm}$ \\
\hline 5. Pantera Rosa $\mathrm{F}_{1}$ con diámetro ecuatorial de bulbillo de $2.0 \mathrm{~cm}$ a menos de $2.5 \mathrm{~cm}$ \\
\hline 6. Pantera Rosa $\mathrm{F}_{1}$ con diámetro ecuatorial de bulbillo de $2.5 \mathrm{~cm}$ a menos de $3,0 \mathrm{~cm}$ \\
\hline 7. Sevillana $\mathrm{F}_{1}$ con diámetro ecuatorial de bulbillo de $1.5 \mathrm{~cm}$ a menos de $2.0 \mathrm{~cm}$ \\
\hline 8. Sevillana $\mathrm{F}_{1}$ con diámetro ecuatorial de bulbillo de $2.0 \mathrm{~cm}$ a menos de $2.5 \mathrm{~cm}$ \\
\hline 9. Sevillana $\mathrm{F}_{1}$ con diámetro ecuatorial de bulbillo de $2.5 \mathrm{~cm}$ a menos de $3.0 \mathrm{~cm}$ \\
\hline
\end{tabular}

Se empleó el diseño estadístico bloques completos al azar con arreglo factorial 3x3 (tres híbridos y tres diámetros ecuatoriales de bulbillo) con 9 tratamientos y tres repeticiones. Se efectúo la prueba estadística de F (ANVA) y prueba de comparación de promedios de Duncan con un nivel de confianza de $\alpha=0,05$.

\section{Evaluaciones}

Las evaluaciones se realizaron a los 80 días después de la plantación (DDP); se evaluó la altura de parte aérea desde la base hasta el ápice de la hoja más larga y el diámetro ecuatorial del bulbo cosechado; se pesó los bulbos comerciales de las categorías "primera" (se consideró un diámetro ecuatorial de 7 a $9 \mathrm{~cm}$ ) y "segunda" (se consideró un diámetro ecuatorial de $5 \mathrm{a} 7 \mathrm{~cm}$ ); y de los bulbos enteros y partidos, para calcular los rendimientos.

Los objetivos del presente trabajo fueron el de determinar el híbrido con mejor comportamiento, el diámetro ecuatorial adecuado de bulbillo y la mejor combinación entre híbrido y diámetro ecuatorial de bulbillo, para la producción de bulbos de cebolla.

\section{RESULTADOS}

A continuación, se muestran el Análisis e varianza para las variables evaluadas así como los efectos principales y efectos simples. 


\section{Tabla 2.}

F calculado $(\alpha=0.05)$ y significancia para las variables evaluadas

\begin{tabular}{|c|c|c|c|c|c|c|c|c|c|c|c|c|c|c|c|c|c|}
\hline \multirow{2}{*}{ FV. } & \multirow{2}{*}{ GL. } & \multicolumn{2}{|c|}{ Alturadplanta } & \multicolumn{2}{|c|}{ Dámetro d bullo 80 DDT } & \multicolumn{2}{|c|}{ Rdto. omercial } & \multicolumn{2}{|c|}{ Rido. caliddd"ppimera" } & \multicolumn{2}{|c|}{ Rito caliddal "segnd" } & \multicolumn{2}{|c|}{ Peso promedio ballo } & \multicolumn{2}{|c|}{ \%BAllosos" partidids } & \multicolumn{2}{|c|}{ Rita. billose enteros } \\
\hline & & F.C. & $\mathrm{Sg}$ & F.C. & $\mathrm{gg}$ & F.C. & $\mathrm{Sg}$ & F.C. & $\mathrm{Sg}$ & F.C. & $\mathrm{Sg}$ & F.C & $\mathrm{Sg}$ & F.C. & $\mathrm{Sg}$ & F.C & $\mathrm{Sg}$ \\
\hline Bloqe & 2 & 3.56 & NS & 1.3 & NS & 0.01 & NS & 0.4 & NS & 1.73 & NS & 4.93 & * & 1.01 & NS & 0.63 & * \\
\hline Hithich & 2 & 8.17 & $*$ & 6.05 & 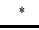 & 31.66 & 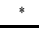 & 8.01 & $*$ & 9.54 & * & 3573 & $*$ & 9.99 & $*$ & 8.34 & * \\
\hline DEB & 2 & 1.92 & NS & 2.61 & NS & 0.15 & NS & 1.55 & NS & 8.92 & NS & 50.31 & * & 988.54 & * & 11899 & * \\
\hline Hilinibx $\mathrm{DEB}$ & 4 & 2.61 & NS & 1.81 & NS & 0.04 & NS & 0.85 & NS & 2.79 & NS & 2.38 & NS & 9.04 & $*$ & 4.57 & * \\
\hline Eroverp. & 16 & & & & & & & & & & & & & & & & \\
\hline Total & 26 & & & & & & & & & & & & & & & & \\
\hline CV.(\%) & & 3.1 & & 9.51 & & 11.64 & & 20.89 & & 26.89 & & 10.02 & & 5.07 & & 23.79 & \\
\hline
\end{tabular}

* Datos obtenidos mediante transformación angular

En la tabla 2 se observa que existe diferencia estadística significativa en la fuente de variación hibrido para todas las variables evaluadas asimismo se observa que en la fuente de variación DEB existe diferencia estadística significativa para peso promedio de bulbo, $\%$ de bulbos partidos y rendimiento de bulbos enteros. Para la interacción hibrido x DEB existe diferencia estadística significativa para \% de bulbos partidos y rendimiento de bulbos enteros.

\section{Efectos Principales para las Variables Evaluadas}

En las tablas 3 y 4 se presentan los efectos principales para las variables evaluadas acorde a la información obtenida en la tabla 2 .

\section{Tabla 3.}

Efecto principal del factor Hibrido en promedio de los niveles del factor DEB

\begin{tabular}{|c|c|c|c|c|c|c|c|c|}
\hline Hibrido & $\begin{array}{l}\text { Altura parte } \\
\text { aérea }(\mathrm{cm})\end{array}$ & $\begin{array}{l}\text { Diámetro bulbo } \\
80 \text { DDT }(\mathrm{cm})\end{array}$ & $\begin{array}{l}\text { Rdto. comercial } \\
\text { t ha }^{-1}\end{array}$ & $\begin{array}{l}\text { Rdto. Categoría } \\
\text { "primera" t } \text { ha }^{-1}\end{array}$ & $\begin{array}{l}\text { Rdto. Categoría } \\
\text { "segunda" t ha } \\
1\end{array}$ & $\begin{array}{l}\text { Peso promedio } \\
\text { bulbo }(\mathrm{g})\end{array}$ & $\begin{array}{l}\text { Bulbos } \\
\text { partidos (\%) }\end{array}$ & $\mid \begin{array}{l}\text { Rdto. Bulbos } \\
\text { enteros } \mathrm{t} \mathrm{ha}^{-1}\end{array}$ \\
\hline Sivan $F_{1}$ & $74.13 \mathrm{a}$ & $6.13 \mathrm{a}$ & $100.44 \mathrm{a}$ & $71.51 \mathrm{a}$ & $28.93 \mathrm{a}$ & $0.18 \mathrm{a}$ & $49.02 \mathrm{a}$ & $39.08 \mathrm{a}$ \\
\hline $\begin{array}{ll}\text { Pantera } & \text { Rosa } \\
F_{1} & \\
\end{array}$ & $73.37 \mathrm{a}$ & $5.38 \mathrm{~b}$ & $90.45 \mathrm{~b}$ & $62.94 \mathrm{a}$ & $27.51 \mathrm{a}$ & $0.17 \mathrm{a}$ & $47.88 \mathrm{a}$ & $32.23 \mathrm{a}$ \\
\hline Sevillana $F_{1}$ & $70.10 \mathrm{~b}$ & $5.36 \mathrm{~b}$ & $64.46 \mathrm{c}$ & $47.84 \mathrm{~b}$ & $16.61 \mathrm{~b}$ & $0.12 \mathrm{~b}$ & $42.17 \mathrm{~b}$ & $24.47 \mathrm{~b}$ \\
\hline
\end{tabular}

*Promedios seguidos con letras iguales no son diferentes estadísticamente (Duncan al 0,05).

En la tabla 3 se observa que para todas las variables evaluadas a excepción de diámetro de bulbo y rendimiento comercial, los híbridos Sivan $F_{1}$ y Pantera Rosa $F_{1}$ muestran ser estadísticamente iguales entre si y diferentes del hibrido Sevillana $\mathrm{F}_{1}$. Para la variable diámetro de bulbo los híbridos Pantera Rosa $F_{1}$ y Sevillana $F_{1}$ muestran ser estadísticamente iguales entre si y diferentes del hibrido Sivan $F_{1}$. Para la variable rendimiento comercial existen diferencias estadísticas significativas entre los tres híbridos siendo el hibrido Sivan $\mathrm{F}_{1}$ el que mostro mayor rendimiento.

\section{Tabla 4.}

Efecto principal del factor DEB en promedio de los niveles del factor híbrido.

\begin{tabular}{|c|c|c|c|}
\hline$D E B$ & $\begin{array}{l}\text { P esop rom e } \\
\text { bu } 1 \text { b o (g) }\end{array}$ & \begin{tabular}{|l|} 
B u lbos \\
p artidos
\end{tabular} & \begin{tabular}{|l|} 
R d to. B u $1 b$ \\
enteros $\dot{t}^{1} h$ \\
\end{tabular} \\
\hline $\begin{array}{l}1.5 \mathrm{a} \mathrm{m} \text { en ods } \\
2.0 \mathrm{~cm}\end{array}$ & $0.20 \mathrm{a}$ & $13.74 c$ & 62.46 a \\
\hline $\begin{array}{l}2.0 a \mathrm{~m} \mathrm{e} \mathrm{n} \mathrm{odse} \\
2.5 \mathrm{c} \mathrm{m} \\
\end{array}$ & $0.14 \mathrm{~b}$ & $40.93 b$ & $24.61 \mathrm{~b}$ \\
\hline $\begin{array}{l}2.5 \mathrm{a} \mathrm{m} \text { e } \mathrm{n} \text { ods } \\
3.0 \mathrm{~cm}\end{array}$ & $0.13 \mathrm{~b}$ & 84.39 a & 8.71 \\
\hline
\end{tabular}

*Promedios seguidos con letras iguales no son diferentes estadísticamente (Duncan al 0,05).

En la tabla 4 se observa que para las variables \% de bulbos partidos y rendimiento de bulbos enteros, existen diferencias estadísticas significativas entre los tres DEB obteniéndose los mayores \% de bulbos partidos con el DEB 2,5 a menos de 3,0 cm y por consiguiente el mayor rendimiento con el DEB 1,5 a menos de 2,0 cm. Para la variable peso promedio de bulbo el DEB 1,5 a menos de 2,0 cm mostro ser estadísticamente diferente de los otros DEB y estos mostraron estadísticamente iguales, obteniéndose 
los mayores \% de bulbos partidos con el DEB 2,5 a menos de 3,0 cm y por consiguiente el mayor rendimiento con el DEB 1,5 a menos de 2,0 cm. Para la variable peso promedio de bulbo el DEB 1,5 a menos de 2,0 cm mostro ser estadísticamente diferente de los otros DEB y estos mostraron estadísticamente iguales, obteniéndose el mayor peso promedio e bulbo con el DEB 1,5 a menos de 2,0 $\mathrm{cm}$.

\section{Efectos Simples para las Variables Evaluadas}

En las tablas 5 y 6 se presentan los efectos simples para las variables evaluadas acorde a la información obtenida en la tabla 2.

\section{Tabla 5.}

Efecto simple del factor DEB en los niveles del factor Híbrido

\begin{tabular}{|l|l|l|l|l|l|l|}
\cline { 2 - 7 } \multicolumn{1}{c|}{} & \multicolumn{3}{c|}{ \% Bulbos partidos } & \multicolumn{3}{l|}{ Rendimiento de bulbos enteros t ha-1 } \\
\hline DEB & Sivan & Pantera rosa & Sevillana & Sivan & Pantera rosa & Sevillana \\
\hline $\begin{array}{l}2.5 \text { a menos de } \\
3.0 \mathrm{~cm}\end{array}$ & $82.00 \mathrm{a}$ & $86.40 \mathrm{a}$ & $84.57 \mathrm{a}$ & $6.82 \mathrm{c}$ & $12.73 \mathrm{c}$ & $6.57 \mathrm{c}$ \\
\hline $\begin{array}{l}2.0 \mathrm{a} \text { menos de } \\
2.5 \mathrm{~cm}\end{array}$ & $50.77 \mathrm{~b}$ & $42.47 \mathrm{~b}$ & $29.57 \mathrm{~b}$ & $30.70 \mathrm{~b}$ & $30.10 \mathrm{~b}$ & $13.03 \mathrm{~b}$ \\
\hline $\begin{array}{l}1.5 \mathrm{a} \text { menos de } \\
2.0 \mathrm{~cm}\end{array}$ & $14.10 \mathrm{c}$ & $14.77 \mathrm{c}$ & $12.37 \mathrm{c}$ & $79.72 \mathrm{a}$ & $53.87 \mathrm{a}$ & $53.80 \mathrm{a}$ \\
\hline
\end{tabular}

*Promedios seguidos con letras iguales no son diferentes estadísticamente (Duncan al 0,05).

En la tabla 5 se observa que para la variable \% de bulbos partidos para los 3 híbridos evaluados existe diferencia estadística significativa entre ellos para los tres DEB, siendo el DEB 2,5 a menos de 3,0 cm el que mostro mayor \%. Para la variable rendimiento de bulbos enteros para los 3 híbridos evaluados existe diferencia estadística significativa entre ellos para los tres DEB, siendo el DEB 1,5 a menos de 2,0 el que obtuvo el mayor rendimiento.

Tabla 6.

Efecto simple del factor híbrido en los niveles del factor DEB.

\begin{tabular}{|l|c|c|l|}
\cline { 2 - 4 } \multicolumn{1}{c|}{} & \%Bulbos partidos & \multicolumn{2}{l|}{ Rendimientodebulbosenteros } \\
\hline Hibrido & 20amenosde25cm & 15amenosde20cm & 20amenosde25cm \\
\hline Sivan F1 & $50.77 \mathrm{a}$ & $79.72 \mathrm{a}$ & $30.70 \mathrm{a}$ \\
\hline Panterarosa F1 & $4247 \mathrm{~b}$ & $53.87 \mathrm{~b}$ & $30.10 \mathrm{a}$ \\
\hline Seillana F1 & 29.57c & $53.80 \mathrm{~b}$ & $13.0 \mathrm{~b}$ \\
\hline
\end{tabular}

*Promedios seguidos con letras iguales no son diferentes estadísticamente (Duncan al 0,05).

En la tabla 6 se observa que para la variable \% de bulbos partidos los tres híbridos evaluados existe diferencia estadística significativa entre ellos para el DEB 2,0 a menos de 2,5 cm siendo el hibrido Sivan $\mathrm{F}_{1}$ el que obtuvo el mayor \%. Para la variable rendimiento de bulbos enteros el hibrido Sivan mostro ser estadísticamente diferente a los demás híbridos y estos iguales estadísticamente entre sí para el DEB 1,5 a menos de 2,0 cm siendo este híbrido el que obtuvo el mayor rendimiento de bulbos enteros, $\sin$ embargo, para el diámetro 2,0 a menos de 2,5 $\mathrm{cm}$ los híbridos Sivan $\mathrm{F}_{1}$ y Pantera rosa $\mathrm{F}_{1}$ mostraron ser 
estadísticamente iguales entre si y diferentes del híbrido Sevillana $\mathrm{F}_{1}$.

\section{DISCUSIÓN}

Con relación al número de hojas y la altura de parte aérea, la diferencia encontrada para el factor cultivar se explica por la composición genética y expresión génica de cada cultivar. Así Sivan $F_{1}$ y Pantera Rosa $F_{1}$ tienen un mayor número de hojas y una mayor altura de parte aérea que Sevillana $\mathrm{F}_{1}$, lo que puede conllevar a una mayor área fotosintética y por ende una mayor fotosíntesis que se puede traducir en bulbos de mayor tamaño; en cambio los diámetros de bulbillos no muestran diferencias probablemente debido a que no hay efecto de la cantidad de energía de los bulbillos para con el numero de hojas, y de igual manera para la altura de la parte aérea después del brotamiento las nuevas plantas tuvieron igual oportunidad de crecimiento. Los efectos genéticos y ambientales en la producción de cebolla provenientes de bulbillos son reportados por Khokhar (2017) y por Ombodi (2013), además que un exceso de nitrógeno puede generar enviciamiento (Khokhar, 2017). Para el diámetro del bulbo Sivan $\mathrm{F}_{1}$ tiene mayor diámetro ecuatorial que Pantera Rosa $F_{1}$ y Sevillana $F_{1}$, principalmente por su condición genética y por el vigor hibrido superior a los otros híbridos, siendo también una consecuencia de un mayor número de hojas y mayor altura de parte aérea. El rendimiento comercial obtenido en general tanto para el factor hibrido como para el diámetro ecuatorial del bulbillo los rendimientos son altos, por encima de $63 \mathrm{t} \mathrm{ha}^{-1}$, comparado con los rendimientos comerciales (provenientes de bulbillos) normalmente alcanzados en cebollas rojas como la 'Roja Camaneja' de polinización abierta con 30 a $40 \mathrm{t} \mathrm{ha}^{-1}$, e incluso superior al rendimiento promedio para la cebolla roja en general en la región Arequipa con $46 \mathrm{t} \mathrm{ha}^{-1}$ (Gobierno Regional de Arequipa, 2018). En el caso de los híbridos el alto rendimiento de Sivan
$F_{1}\left(100,4 t^{-1} a^{-1}\right)$ y Pantera Rosa $F_{1}\left(90,5 t a^{-1}\right)$ puede explicarse por la capacidad de aclimatación de los híbridos, el vigor hibrido, y su potencial genético, el cual se vio reflejado con un mayor número de hojas y una mayor altura de la parte aérea. El hibrido Sevillana $\mathrm{F}_{1}$, si bien su rendimiento es menor $\left(64,8 \mathrm{tha}^{-1}\right)$, también es un rendimiento alto. Los rendimientos encontrados para Sivan $F_{1}$ y Pantera Rosa $F_{1}$ son muy superiores a los reportados para Sivan $F_{1}$ por Armejo (2006); para 'Roja Camaneja' por Ninasivincha (2008); y para 'Roja Americana' por Málaga (1992), a nivel nacional. A nivel internacional se reportan rendimientos también inferiores como el caso de Ketema et al. 2013, Madisa (1994); Khokhar et al. (2002).

El rendimiento en el factor diámetro ecuatorial de los bulbillos no se observa una mayor diferencia, siendo ligeramente superior en los bulbillos de 2.0 a menos de $2.5 \mathrm{~cm}$. Al analizar el rendimiento de la cebolla de "primera", persiste el mayor rendimiento de Sivan $F_{1}$ y Pantera Rosa $\mathrm{F}_{1}$, superior a Sevillana $\mathrm{F}_{1}$; y en los bulbillos la tendencia es que a menor diámetro de bulbillo mayor rendimiento; mientras que en caso de la cebolla de "segunda" se mantiene la superioridad de Sivan $\mathrm{F}_{1}$ y Pantera Rosa $\mathrm{F}_{1}$, pero para el caso de los bulbillos la cantidad es mayor a medida que el bulbillo aumenta de diámetro. El análisis del peso promedio del bulbo cosechado muestra que Sivan $\mathrm{F}_{1}$ y Pantera Rosa $\mathrm{F}_{1}$, son superiores a Sevillana $F_{1}$, lo que influye significativamente en el rendimiento comercialy de "primera". De la misma forma hay superioridad de los diámetros de bulbillo de $1.2 \mathrm{a}$ menos de $2.0 \mathrm{~cm}$ sobre los otros diámetros, lo que se refleja principalmente en el rendimiento de "primera". Al analizar el porcentaje de bulbos enteros y bulbos partidos se halló que no hay mucha diferencia para el factor híbrido, aunque de Sivan $\mathrm{F}_{1}$ tiene una mayor proporción de bulbos enteros que Pantera Rosa $F_{1}$ y Sevillana $F_{1}$, siendo lo contrario para el porcentaje de bulbos partidos. Diversas investigaciones (Ketema et al. 
2013; Armejo, 2006; Ninasivincha, 2008; Tamo, 2010; Khokhar et al. (2002) muestran la presencia de bulbos múltiples o partidos en diferentes cantidades, especialmente cuando los bulbillos son mayores de 2,0 $\mathrm{cm}$ de diámetro ecuatorial. La presencia de bulbos múltiples es frecuente en las plantas provenientes de bulbillos mayores a 2,0 cm y los bulbos formados alcanzan los diámetros comerciales de primera o segunda y están considerados dentro de los bulbos comerciales y se venden en el mercado como bulbos de primera. Al parecer en los bulbillos grandes ya se tiene formadas yemas laterales que son las que durante el crecimiento de la planta se separan y forman bulbos múltiples; lo que no estaría ocurriendo u ocurre muy poco con las plantas provenientes de bulbillos menores a 2,0 $\mathrm{cm}$. Cuando se analiza la producción de bulbos enteros se halló que las cantidades son muy bajas para los bulbillos de más de $2,0 \mathrm{~cm}$ y muy altas para los diámetros menores a $2,0 \mathrm{~cm}$, independiente del hibrido. En el caso del tratamiento Sivan $F_{1}$ con bulbillos de 1,5 a menos de 2,0 cm de diámetro el rendimiento de bulbos enteros fue de 79,7 $\mathrm{t} \mathrm{ha}^{-1}$, con un porcentaje de bulbos enteros de $84,6 \%$, un rendimiento de primera de $81,3 \mathrm{tha}^{-1}$, un rendimiento de segunda de $17,1 \mathrm{tha}^{-1}$ y un rendimiento comercial de $98,4 \mathrm{t}$ $\mathrm{ha}^{-1}$. En general los resultados encontrados concuerdan con lo señalado por varios autores sobre la factibilidad de uso de bulbillos para la producción comercial de bulbos de cebolla como Jordaan et al. (2017), y en Arequipa, por Málaga (1992), Armejo (2006), Ninasivincha (2008) y Tamo (2010); y que los diámetros ecuatoriales de bulbillo óptimos son de $25 \mathrm{~mm}$ (Serra y Currah, 2002; y Brewster, 2008); entre 8 y $21 \mathrm{~mm}$ (O'Connor, 2006); $2 \mathrm{~cm}$ (Singh et al., 1996); de 1,9 a 2,5 cm (Madisa, 1994); 1,7 cm (Khokhar et al., 2002); así como mayor precocidad (Ketema et al., 2013). La ausencia de plantas con floreamiento prematuro o bolting, puede deberse a que el almacenamiento de los bulbillos antes de la plantación ocurrió bajo condiciones de temperaturas altas propias de los meses de octubre, noviembre y diciembre en Arequipa y concuerda con lo señalado por Khokhar et al. (2002), Khokhar et al. (2007) y Khokhar (2017) sobre un mayor rendimiento y menor bolting o que los híbridos estudiados sean tolerantes al frio.

\section{CONCLUSIONES}

- Los híbridos Sivan $F_{1}$ y Pantera Rosa $F_{1}$ tuvieron los mejores rendimientos comerciales, de "primera", de bulbos enteros; mayor pso promedio de bulbo y el menor porcentaje de bulbos partidos o múltiples.

- Los bulbillos con diámetro ecuatorial de 1,5 a menos de $2,0 \mathrm{~cm}$ de diámetro ecuatorial produjeron los mejores pesos promedios de bulbos, el menor porcentaje de bulbos partidos o múltiples, el mayor rendimiento de "primera" y el mayor rendimiento de bulbos enteros.

- La mejor combinación hibrido por diámetro de bulbillo correspondió a Sivan $\mathrm{F}_{1}$ con bulbillos de 1,5 a menos de 2,0 cm de diámetro ecuatorial, con una rentabilidad de $64,9 \%$.

\section{BIBLIOGRAFÍA}

Alemzadeh, N. (2007). Effect of density, cultivars and sowing date on onion sets production. Asian Journal for Scientific Information 6(7): 1147-1150.

Armejo, J.A. (2006). Época de siembra y diámetros de bulbillos para la producción de cebolla roja (Allium cepa L.) híbrido Sivan. Tesis Ingeniero Agrónomo. Universidad Nacional de San Agustín de Arequipa. Arequipa, Perú.

Brewster, J.L. (2008). Onion and other alliums. 2nd edition. Wallingford: CAB International. $432 \mathrm{p}$.

Gobierno Regional de Arequipa (GRA). 2018. Estadística agrícola. Gerencia Regional de Agricultura. 
Jordaan, M.; Coetzer, G.M.; Allemann, J. (2017). Potential of Adelaide, Cradock and Middelburg, South Africa, for out-ofseason onion production using different planting materials and planting dates. South AfricanJournal of Plant and Soil 34: 1-8.

Ketema, S.; Dessalegn, L.;\&Tesfaye, B. (2013). Effect of planting methods on maturity and yield of onion (Allium cepa var cepa) in the Central Rift Valley of Ethiopia. Ethiop. J. Agric. Sci. 24:45-55.

Kivijarvil, P.; Hannukkala, A.; Haapalainen, M.;\&Iivonen, S. (2017). Onion seedlings versus onion sets in organic onion production. $4^{\text {th }}$ NJF Organic Conference NJF 495 seminar. Natural Resources Institute Finland.

Khokhar, K.M.; Hussain, S.I.; Mahmood, T.; \& Laghari, M.H. (2002). Bulb yield and quality as affected by set size in autumn season onion crop. Asian Journal of Plant Sciences 1 (6): 657-758.

Khokhar, K.M.; Hadley, P.; \&Pearson, S. (2007). Effect of cold temperature durations of onion sets in store on the incidence of bolting, bulbing and seed yield. Scientia Horticulturae 112 (1):16-22.

Khokhar, K.M.(2017). Environmental and genotype effects on bulb development in onion - a review. The Journal of Horticultural Science and Biotechnology, Volume 92: 448-454.

Köycü, N.D.; Özer, N. (1997). Determination of seedborne fungi in onion and their transmission to onion sets. Phtytoparasitica 25:5

Madissa, M.E. (1994). The effect of planting date, set size, and spacing on the yield of onion (Allium cepa) in Botswana. ISHS ActaHorticulturae 358.

Málaga, R.E. (1992). Producción de cebolla (Allium cepa L.) 'Roja Americana' propagada por bulbillos y elaboración de compost. Informe de prácticas preprofesionales para optar el grado académico de bachiller en Ciencias Agrarias. Universidad Nacional de San Agustín de Arequipa. Arequipa-Perú.

Ninasivincha, Y.M. (2008). Efecto del nitrógeno y diámetro de bulbillos en el rendimiento de cebolla (Allium cepa L.) cv. 'Roja Camaneja'-Camana 2006. Tesis ingeniero agrónomo. Universidad Nacional de San Agustín de Arequipa. Arequipa-Perú.

O'Connor, D. (2006). Production of onion sets and onions from sets. Vegetable Crops Research Bulletin Vol. 64: 181-187.

Ombódi, A.; Koczka, N.; Lugasi, A.; Daood, H.G.; Berki, M.; \& Helyes, L. (2013). Nutritive constituents of onion grown from sets as affected by water supply. Hortscience 48(12): 1543-1547.

Peluffo, S.; Gonzáles.; Borges, A.; Arboleya, J.; \& Galván, G.A. (2016). Onion sets as planting material for seed production of three cultivar en Uruguay. Seed Science and Technology Volume 44, number 3:500-513.

Saraswathi, T.; Sathiyamurthy, V.A.; Tamilselvi, N.A.; \& S. Harish,S. (2017). Review on Aggregatum onion (Allium cepa L. var. aggregatum Don.).International Journal of Current Microbiology and Applied Sciences Volume 6 Number 4:1649-1667.

Sekara, A; Pokluda R; Del Vacchio L; Somma S; \& Caruso G.(2017).Interactions among genotype, environment and agronomic practices on production and quality of storage onion (Allium cepa L.) - a review. Hort. Sci. (Prague) 44 (1): 21-42.

Serra, A.D.B. \& Currah, L. (2002). Agronomy of onions, p. 187-232. In: Rabinovitch, H.D. \& Currah, L. (eds.). Allium crop science: Recent advances. CABI, Wallingford, UK. Singh, B.; Babel, Y.S.; Yadav, B.D.; \&Sharma, D.R. (1996). Effect of size onion sets and planting distance on yield and 
economics of kharif onion. Indian Journal of Horticulture 62(3): 215-217.

Tamo, J. (2010). La influencia del nitrógeno en el diámetro de bulbito en el rendimiento de cebolla (Allium cepa L.) cv. "Roja Camaneja" para la campaña de otoño en el valle de Camaná. Tesis para optar el grado de Magister Scientiae. UNALM. LimaPerú. 118 pp.

\section{CORRESPONDENCIA}

Alberto Anculle-Arenas

Urbanización Aurora S/N,Arequipa, Perú.

albertoanculle@gmail.com 